\title{
An exploratory investigation of the effect of naturalistic light on fatigue and subjective sleep quality in stroke patients admitted for rehabilitation: A randomized controlled trial
}

\author{
Anders West ${ }^{\mathrm{a}, *}$, Sofie Amalie Simonsen ${ }^{\mathrm{a}}$, Poul Jennum ${ }^{\mathrm{b}}$, Niklas Cyril Hansen ${ }^{\mathrm{a}}$, Marie Schønsted ${ }^{\mathrm{a}}$, \\ Alexander Zielinski ${ }^{\mathrm{a}}$, Birgit Sander ${ }^{\mathrm{c}}$ and Helle K. Iversen ${ }^{\mathrm{a}}$ \\ ${ }^{a}$ Clinical Stroke Research Unit, Department of Neurology, Rigshospitalet, Faculty of Health Sciences, \\ University of Copenhagen, Copenhagen, Denmark \\ ${ }^{\mathrm{b}}$ Danish Center for Sleep Medicine, Department of Neurophysiology Rigshospitalet, Faculty of Health Sciences, \\ University of Copenhagen, Copenhagen, Denmark \\ ${ }^{\mathrm{c}}$ Department of Ophthalmology, Rigshospitalet, Copenhagen University Hospital, Copenhagen, Denmark
}

\begin{abstract}
.
BACKGROUND: Daylight entrains the central circadian pacemaker to the 24-hour day and is crucial for optimal alertness and sleep-quality. Rehabilitation patients tend to lack exposure to sufficient natural light.

OBJECTIVE: Installed diurnal naturalistic light may reduce the known disrupted sleep quality and fatigue seen in post stroke patients.

METHODS: Stroke patients were randomized to either an intervention rehabilitation unit (IU) equipped with naturalistic lighting (artificial sunlight spectrum) or to a control rehabilitation unit (CU) with standard indoor lighting. At inclusion and discharge, fatigue and subjective sleep quality were measured.

RESULTS: Ninety stroke patients were included between May 2014, and June 2015. At discharge, patients from the IU experienced less fatigue than the $\mathrm{CU}$ patients, based on the Multidimensional Fatigue Inventory questionnaire general (IU, $n=28$; CU, $n=30$; diff $-20.6 \%, 95 \%$ confidence interval (CI) $[-35.0 \% ;-3.0 \%] ; P=0.025$ ) and the Rested Statement (IU, $n=28 ; \mathrm{CU}, n=30 ;$ diff $+41.6 \%, 95 \%$ CI $[+4.6 \% ;+91.8 \%] ; P=0.025)$. No differences were detected between groups in sleepiness or subjective sleep quality by the Pittsburgh Sleep Quality Index.
\end{abstract}

CONCLUSIONS: Fatigue was significantly reduced in rehabilitation patients exposed to naturalistic lighting during admission.

Keywords: Sleep, fatigue, circadian rhythm, clinical trials, stroke, light

\section{Introduction}

Stroke is a leading cause of death and disability, with high costs for patients and their families,

\footnotetext{
*Address for correspondence: Anders Sode West, MD, PhD., Clinical Stroke Research Unit, N25, Department of Neurology, Rigshospitalet, Glostrup, Copenhagen 2600, Denmark. Tel.: +45 217485 87; E-mail: anders.sode.west@ regionh.dk.
}

as well as for society (Feigin et al., 2015; Jennum, Iversen, Ibsen, \& Kjellberg, 2015). The combination of immobilization and long-term hospitalization is often associated with a lack of natural light during the day and input of artificial light from the hospital indoor lighting in the evening and night. The intrinsically photosensitive retinal ganglion cells absorb light and are connected by the retinohypothalamic 
tract to the master circadian clock system located in the suprachiasmatic nucleus. This constellation makes light, especially the short-wavelength blue light (Thapan, Arendt, \& Skene, 2001), the strongest Zeitgeber (time giver) for the circadian rhythm and the sleep-wake cycle.

Compared to the duration of prior wakefulness, the endogenous circadian rhythm plays a significant role in controlling the length of sleep and its timing system. Using bright light exposure may control this and even consolidate the sleep, but further studies are needed investigating the circadian effect on sleep quality (Ancoli-Israel et al., 2003; Czeisler, Weitzman, Moore-Ede, Zimmerman, \& Knauer, 1980). Sleep disturbances during hospitalization have been described, including in stroke patients, who often also suffer from multiple co-morbidities (Hermann, 2004; Venkateshiah, 2012).

Despite this understanding, only six studies, investigating the influence of light on sleep in a real-life hospital setting (delirium patients were not included), could be identified in a PubMed search. Three of these studies yielded positive findings regarding sleep time, time in bed, and wakefulness (Mishima et al., 1994; Satlin, Volicer, Ross, Herz, \& Campbell, 1992; Wakamura \& Tokura, 2001). In contrast, the other three studies had neutral findings regarding sleep time and sleep quality (De Rui et al., 2014; Kobayashi et al., 2001; Perras, Meier, \& Dodt, 2007). Small sample sizes and no control groups characterized all six studies. However, it seems that low light intensity may be correlated with negative consequences for sleep and fatigue in hospitalized patients (Bernhofer, Higgins, Daly, Burant, \& Hornick, 2013; Cho, Joo, Koo, \& Hong, 2013).

Disrupted sleep, characterized by shorter sleep duration during the night and greater daytime sleepiness has been related to reduced physical and cognitive performance (Goldman et al., 2007). Sleep itself and decreased daytime sleeping may however improve motor consolidation after physical training (Siengsukon \& Boyd, 2009) and the cognitive function after discharge (Dzierzewski et al., 2014). Prevention of sleep disturbances and improvement in sleep quality may therefore be necessary to achieve optimal rehabilitation outcome after stroke.

Fatigue often co-exists with sleep disturbances, especially in healthy people; however, fatigue is also a frequent pathophysiological consequence of severe diseases and therefore not necessarily related to sleepiness and/or sleep quality. To our best knowledge, no scientific distinct separation can be explain between sleepiness and fatigue since the pathophysiology of fatigue is not fully understood, but changes in the biochemical environment in the brain as a sequelae after stroke may be implicated in the development of post-stroke fatigue (De Doncker, Dantzer, Ormstad, \& Kuppuswamy, 2017). The main behavioral consequence of fatigue is a significant reduction in self-initiated voluntary behavior and sleepiness is related to increased sleep pressure due to the activity of ventrolateral preoptic nucleus and its related areas because of insufficient sleep (Lu, Greco, Shiromani, \& Saper, 2000).

Post-stroke fatigue is common and affects approximately $25 \%$ to $85 \%$ of patients (Cumming, Packer, Kramer, \& English, 2016). One study even found that $40 \%$ of stroke patients ranked fatigue as the worst or one of the worst sequelae of stroke (Ingles, Eskes, \& Phillips, 1999). Post-stroke fatigue is believed to interfere with stroke recovery (Hermann \& Bassetti, 2009) and is correlated with decreased activities of daily living, health-related quality of life after discharge (Chen et al., 2015; van de Port, Kwakkel, Schepers, Heinemans, \& Lindeman, 2007), and mortality (Mead et al., 2011).

No effective treatment has proven to prevent or treat fatigue (Hinkle et al., 2017; Wu et al., 2015), which is often associated with critical diseases that also show circadian disruption (Ancoli-Israel et al., 2014). However, the relationship between fatigue and circadian disruption is still unknown. One study found that 45 minutes of daily blue light exposure reduced daytime fatigue in discharged patients after traumatic brain injury (Sinclair, Ponsford, Taffe, Lockley, \& Rajaratnam, 2014), but to our knowledge, no studies have investigated the effect of naturalistic light or other light interventions on fatigue during hospitalization.

The aim was to study the effect of naturalistic light on fatigue and subjective sleep quality in patients admitted for neurorehabilitation after stroke.

\section{Materials and methods}

\subsection{Study design and participants}

This study is a quasi-randomized, controlled trial and was conducted in the Stroke Unit, Department of Neurology, Rigshospitalet, Copenhagen, Denmark. From May 2014 to June 2015, stroke patients who needed in-hospital neurorehabilitation for more than 2 weeks were recruited from the acute stroke unit 
and randomized into two arms: 1) the intervention unit (IU), with rehabilitation in a unit equipped with naturalistic light; and 2) the control unit (CU), with rehabilitation in a unit with standard indoor lighting. Patients were excluded if they could not give informed consent because of their awareness status, severe aphasia, or if they were expected to be hospitalized in the rehabilitation unit for less than 2 weeks. No safety precautions were necessary regarding assessments and interventions. The study was approved by the Danish scientific ethics committee (H-4-2013-114) and the Danish Data Protection Agency (2007-58-0015). ClinicalTrials.gov Identifier: NCT02186392. A thoroughly detailed methods description has been published elsewhere (West et al., 2017).

\subsection{Randomization}

The included patients were randomized to the IU or $\mathrm{CU}$ by non-blinded stroke nurses (quasirandomization) at the acute stroke unit (with normal standard light conditions). However, the nurses were not involved in the study and were simply following normal procedure regarding the relocation of patients to the two rehabilitation units, based on whether there were available beds.

\subsection{Outcomes}

The study was part of a larger project investigating the effects of naturalistic light on stroke patients measured by psychological parameters, biochemical parameters, fatigue, and sleep. Because it involves a relatively new scientific area, the study was defined as an exploratory investigational study. We therefore chose five primary endpoints which include sleep quality/sleepiness and fatigue.

\subsection{Naturalistic light intervention}

A 24-hour naturalistic lighting scheme was implemented in all areas of the IU using multi-colored light-emitting diode-based luminaires (LED-lamps) along with a centralized lighting controller to manage all luminaires according to the selected lighting scheme (Chromaviso, Denmark). The naturalistic lighting scheme was constantly running during the inclusion period and the luminaires were located in the ceiling and on the wall behind the beds. According to the scheme, the light (light levels at the outer of the eye of a patient lying in the bed) started as dim in the morning with a very low content of blue light at $7 \mathrm{am}(<2700 \mathrm{~K}$ and melanopic irradiance $<0.08 \mu \mathrm{W} / \mathrm{cm}^{2}$ ), increasing to reach maximum illuminance at noon $(6000 \mathrm{~K}$ and melanopic irradiance at $45 \mu \mathrm{W} / \mathrm{cm}^{2}$ ) with a high content of blue light, and then from $2 \mathrm{pm}$ slowly dimming again through the evening, changing to warm white at $6 \mathrm{pm}(3000 \mathrm{~K}$ and melanopic irradiance $<10 \mu \mathrm{W} / \mathrm{cm}^{2}$ ). It continued to dim with minimal blue light content to turn off completely at $10 \mathrm{pm}$ in the patient's rooms whereas it continued as amber colour $(1850 \mathrm{~K}$ and melanopic irrandiance $<0,08 \mu \mathrm{W} / \mathrm{cm}^{2}$ ) in hallways and toilets. If the light was turned on in the patient's rooms in the IU between $10 \mathrm{pm}$ and $7 \mathrm{am}$, it would be with very low blue content $(1850 \mathrm{~K}$ and melanopic irrandiance $<0,08 \mu \mathrm{W} / \mathrm{cm}^{2}$ ). The standard indoor light in the $\mathrm{CU}$ was measured to $3000 \mathrm{~K}$ and melanopic irradiances $<10 \mu \mathrm{W} / \mathrm{cm}^{2}$ between $7 \mathrm{am}$ and $9 \mathrm{pm}$. If the light was turned on in the patient's rooms in the $\mathrm{CU}$ between $9 \mathrm{pm}$ and $7 \mathrm{am}$, it was measured to be dimmed $(3000 \mathrm{~K}$ and melanopic irrandiance $>0,75 \mu \mathrm{W} / \mathrm{cm}^{2}$ ).

The technical lights setup was produced in accordance with CIE TN 003 following the principles of Lucas et al. (Lucas et al., 2014). Due to the complexity and the need for comprehensive technical description of the light, the light intervention is presented in details in the method description paper (West et al., 2017). In the CU, all normal ceiling luminaries had new fluorescent tubes installed prior to the inclusion in order to stabilize the light in all areas of the unit. The technical light description regarding the irradiance profiles for the IU and CU can be found in Fig. 3a and $3 b$ in the method paper (West et al., 2017). Blackout curtains were installed in the IU, and they were raised at 8 am and lowered at $8 \mathrm{pm}$ for all four seasons to avoid incoming light in this time period.

\subsection{Assessments}

\subsubsection{Questionnaires}

The questionnaires were self-administered; however, if the patient had difficulties filling out the questionnaire because of visual or mobile disabilities, the examiner did the reading and writing. The questionnaires were administered at inclusion and discharge (hospital treatment complete/done) in the time interval from 09:00 a.m. to 03:00 p.m. Sleepiness was rated using the Epworth Sleepiness Scale (ESS) and the Visual Analog Scala for sleepiness (VAS sleepiness). 
Fatigue was rated with the Multidimensional Fatigue Inventory questionnaire (MFI-20). Feeling of rest was rated by the statement, "I woke up fresh and rested." The Pittsburgh Sleep Quality Index (PSQI) was used for assessment of subjective sleep quality. Missing data regarding single questions in the questionnaires were replaced by the patient's own response from either the inclusion or discharged questionnaire. Questionnaires with more than three missing questions were excluded. Because missing questions were related to neglected questions, it was not a continuous issue (under $10 \%$ of patients) because other reasons for the missing data (unable to complete the questionnaire) resulted in exclusion.

The MFI-20 covers five areas: general fatigue, physical fatigue, reduced activity, reduced motivation, and mental fatigue. "General fatigue" can by itself be used to estimate a patient's level of fatigue. ESS measures general level of daytime sleepiness or average sleep tendency in daily life (Johns, 1991). The total ESS score ranges from 0 to 24 . VAS contains a line with numbers from 0 to 10 with zero indicating no sleepiness and 10 the worst possible immediate feeling of sleepiness. This was explained to the patients before rating. VAS was used every 4 hours over the course of 24 hours at inclusion and discharge. The diurnal VAS for sleepiness in Danish has not been validated and is not alone a recommended instrument to score sleepiness; thus, it was not used for group comparisons. Sleepiness diurnal patterns were collected to estimate the changes in sleepiness during admission, which could be valuable in understanding the collected data regarding ESS and PSQI.

The feeling of being rested complements the quality of previous sleep, which is why we included the statement, "I woke up fresh and rested," from the wellbeing index (WHO-5) questionnaire (Allgaier et al., 2013). The higher the score, the better the feeling of rest. The statement is thought to be an easy way for the patient to express sleep quality information.

The global score of PSQI indicates subjective sleep quality (zero suggests no difficulties, and 21 indicates severe difficulties in all areas). PSQI has a portion concerning the bed partner with 5 self-rated questions, which is not included in the score system, and this part was excluded in this study.

The Morningness-Eveningness Questionnaire (MEQ) was applied to stratify the participants into morning or evening types. MEQ is validated for the individual diurnal rhythm (Horne \& Ostberg, 1976), and a higher score correlates with the morning type. The MEQ questionnaire was, like the other questionnaires, filled out at inclusion and again at discharge. Several questionnaires regarding depression including the Major Depression Inventory was also used. Results of those are to be published elsewhere.

\subsubsection{Polysomnography}

Patients underwent polysomnography (SOMNOscreen ${ }^{\mathrm{TM}}$ ) according to international standards at inclusion and discharges. Patients were asked to refrain from caffeinated beverages, sleep medicine, and CPAP on the night of the examination. In this paper we only includes sleep apnea as confounder in assessments dealing with sleepiness and fatigue (Hermann \& Bassetti, 2016). Data from the following monitors from the PSG were examined: a pressure sensor for recording nasal flow, thoracic and abdominal effort belts, and a pulse oximeter. We used the American Academy of Sleep Medicine (AASM) manual for scoring respiratory events during sleep (Berry et al., 2012).

\subsection{Medication}

Medication registration was performed at inclusion and discharge. Therefore, if patients were only prescribed these medications in a short interim period between these time points, these drugs were not registered and not included in the statistical analysis. The indication for sleep medicine, antidepressant medication, or/and anxiety medication was assessed by the attending physicians on the ward rounds and was frequently prescribed for apathy, depressive mood and fatigue or a combination of these. The indication for these medications was not based on tests but solely on the physician's assessments.

\subsection{Statistical analysis}

Normally distributed continuous variables are presented as means and standard deviations ( \pm SDs). Categorical variables are presented as number of cases and percentage of occurrence. Differences are expressed with $95 \%$ confidence intervals (CIs), and the deviation of calculated differences is expressed as standard error (SE).

For analyzing differences in the global score for PSQI, ESS, all five groups and the global score in the MFI-20 and the statement "I woke up fresh and rested" between the two units, an analysis of covariance (ANCOVA, SAS) was used to integrate the baseline values from the questionnaires in the 
calculation, thus considering the individual differences. To avoid interaction and confounding effect of sleep medicine (N05C) (PSQI, ESS), antidepressant medicine (N06A) (MFI-20, Rested Statement) and morphine (NO2A) (PSQI, EES), these were integrated into the covariance analysis. Due to the significant difference in inclusion period between the two groups, the length of inclusion was included as a confounder in all analyzes. Because of the repeated measurements (diurnal) of VAS, a mixedmodel analysis (Proc Mixed, SAS) was used to describe the variances/changes among the seven time-points.

Due to the non-normally distributed scores and the skewed linearity data were logarithmically transformed before analysis of covariance. The calculated differences in the mixed-model analysis were backtransformed from $\log 2$ to empirical fractiles and converted to percent difference in scores $\left((\mathrm{x}-1)^{*} 100\right)$. Correlation analyzes were done by regression analysis in $\log$ transformed scores (Proc glm, SAS).

$T$-tests were used to describe differences in basic demographic parameters between groups. All analyses were made in SAS (9.4; SAS Inst. Inc., Cary, NC, USA). A $P<0.05$ was considered significant for all the analyses.

\section{Results}

A total of 256 patients who needed in-hospital neurorehabilitation were screened, and 90 patients met the inclusion criteria and agreed to participate. Because of inability to complete the questionnaires due to severe illness or reduced awareness, there was a dropout and exclusion range of 16-24 patients in the IU and 16-27 patients in CU groups, depending on the questionnaire (Fig. 1), resulting in 71 patients completing the study (44 $(62 \%)$ males and 27 (38\%) females), mean age 73 (range 51-96). The wide exclusion range can be explained by the lack of participation in the VAS and by two participants who could not assess all questionnaires at the IU because of reduced vitality. Patient characteristics are presented in Table 1. The two groups were well balanced, but they differed regarding length of inclusion period ( 45 days in the IU vs. 34 days in the CU; $P=0.02$ ). No significant correlation was found regarding length of inclusion and fatigue, sleepiness, or subjective sleep quality as measured by the questionnaires by linear regression analysis. No other significant differences were found between the two groups.

Because VAS was collected every 4 hours, only 39 patients were willing to participate. Due to the comparability between the participants regarding MEQ

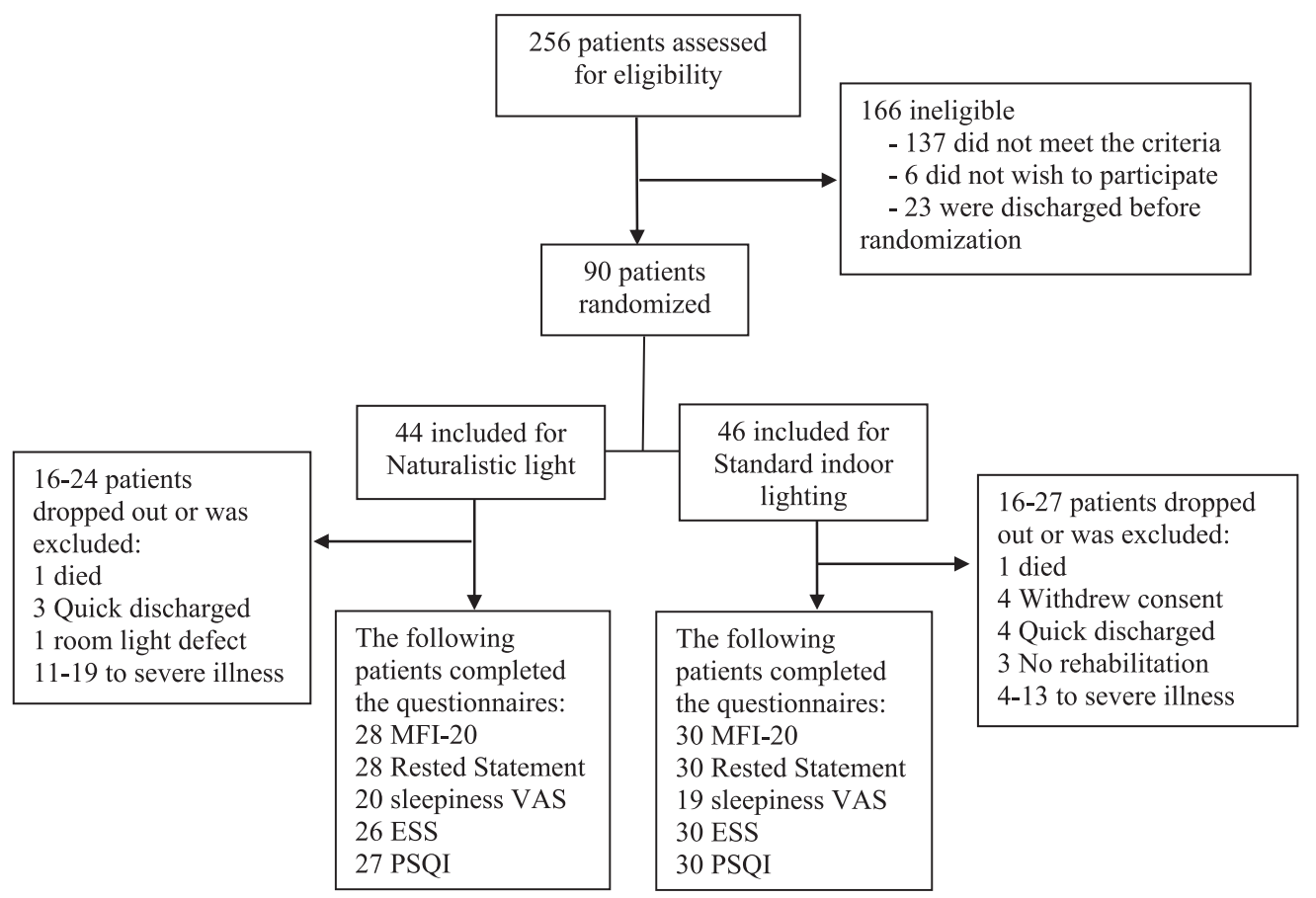

Fig. 1. Trial profile. 
Table 1

Patient characteristics

\begin{tabular}{lccc}
\hline Patient characteristics & $\begin{array}{c}\text { Intervention Unit } \\
(\mathrm{N}=39)\end{array}$ & $\begin{array}{c}\text { Control Unit } \\
(\mathrm{N}=32)\end{array}$ & $P$ value \\
\hline Age, mean years (age interval) & $72.7(55-96)$ & $72.8(51-89)$ & 0.96 \\
Sex, & & & 0.93 \\
$\quad$ Male, $n(\%)$ & $24(62)$ & $20(62)$ & \\
Female, $n(\%)$ & $15(38)$ & $12(38)$ & \\
Time from ictus to inclusion, mean days $( \pm \mathrm{SD})$ & $7.6 \pm 8.3$ & $6.0 \pm 4.4$ & 0.55 \\
Inclusion period, mean days $( \pm \mathrm{SD})$ & $45.3( \pm 22.1)$ & $33.7( \pm 12.7)$ & $\mathbf{0 . 0 2}$ \\
Smoker, $n(\%)$ & $26(67)$ & $26(84)$ & 0.10 \\
Hypertension, $n(\%)^{*}$ & $29(74)$ & $19(59)$ & 0.18 \\
Diabetes & & & \\
Type $1, n(\%)$ & $0(0)$ & $2(6)$ & 0.11 \\
Type $2, n(\%)$ & $7(18)$ & $6(19)$ & 0.93 \\
Hypercholesterolemia, $n(\%)$ & $13(33)$ & $7(22)$ & 0.29 \\
Atrial fibrillation, $n(\%)$ & $5(13)$ & $7(22)$ & 0.31 \\
Depression, $n(\%)^{* *}$ & $2(5)$ & $3(9)$ & 0.49 \\
Barthel, mean score $( \pm \mathrm{SD})$ & $47.6 \pm 34.0$ & $51.7 \pm 28.9$ & 0.65 \\
NIHSS, mean score $( \pm \mathrm{SD})$ & $7.0 \pm 6.4$ & $5.4 \pm 4.2$ & 0.34 \\
MEQ total score, mean $( \pm \mathrm{SD})$ & $60.1 \pm 12.0$ & $60.4 \pm 10.7$ & 0.93 \\
Definitely Evening type, $n(\%)^{* * *}$ & $1(3.7)$ & $0(0)$ & \\
Moderately Evening type, $n(\%)^{* * *}$ & $7(25.9)$ & $5(17.2)$ & \\
Neither type, $\mathrm{n}(\%)^{* * *}$ & $2(7.4)$ & $2(6.9)$ & \\
Moderately Morning type, $n(\%)^{* * *}$ & $10(37.0)$ & $16(55.2)$ & \\
Definitely Morning type, $n(\%)^{* * *}$ & $7(25.9)$ & $6(20.7)$ & \\
\hline
\end{tabular}

*Hypertension defined as under medical treatment for hypertension at the inclusion. ${ }^{* *}$ History of depression. *** Percentage of 27 respondents at IU and 29 at CU. National Institutes of Health Stroke Scale (NIHSS), morningness-eveningness questionnaire (MEQ).

Table 2

Differences in scores between the IU and CU groups

\begin{tabular}{lcccc}
\hline Subjective sleep quality & Difference in favor of IU & Standard error & $95 \%$ CL & $P$ value \\
\hline PSQI $^{\mathrm{a}}$ & $-13.0 \%$ & 0.2 & $-37.1 \% ;+20.5 \%$ & 0.396 \\
\hline Fatigue and sleepiness & Difference in favor of IU & Standard error & $95 \%$ CL & $P$ value \\
\hline ESS $^{\text {a }}$ & $-7.9 \%$ & 0.3 & $-35.0 \% ;+30.5 \%$ & 0.639 \\
Rested Statement $^{\mathrm{b}}$ & $+41.6 \%$ & 0.2 & $+4.6 \% ;+91.8 \%$ & $\mathbf{0 . 0 2 5}^{*}$ \\
MFI-20 global $^{\mathrm{b}}$ & $-10.8 \%$ & 0.1 & $-21.9 \% ;+1.8 \%$ & 0.088 \\
Activity part & $-10.2 \%$ & 0.2 & $-27.1 \% ;+10.7 \%$ & 0.308 \\
General part & $-20.6 \%$ & 0.1 & $-35.0 \% ;-3.0 \%$ & $\mathbf{0 . 0 2 5}$ \\
Mental part & $-6.8 \%$ & 0.1 & $-21.3 \% ;+10.3 \%$ & 0.405 \\
Motivation part & $-4.7 \%$ & 0.2 & $-25.5 \% ;+21.9 \%$ & 0.696 \\
Physical part & $-12.8 \%$ & 0.1 & $-28.0 \% ;+5.5 \%$ & 0.156 \\
\hline
\end{tabular}

Difference in scores between the IU and CU groups (IU - CU) was determined from the analysis of covariance. ${ }^{a}$ Covariance analysis with sleep medicine included as a confounder. ${ }^{\mathrm{b}}$ Covariance analysis with antidepressant medication included as a confounder. Length of inclusion was included in all analyzes as a confounder. Due to non-parametric distribution, the scores were $\log 2$ transformed before calculation. The calculated estimates were back-transformed from $\log 2$ to empirical fractiles to achieve parametric distribution, then converted to percent difference in score $\left((\mathrm{x}-1)^{*} 100\right)$. No back-transformation was done on standard error. Outcome specification: PSQI: high scores indicate decreased sleep quality; ESS: high score indicates a high degree of sleepiness; Rested Statement: higher score indicates better feeling of rest; MFI-20: high scores indicate a high degree of fatigue.

subtypes (Table 1), the MEQ results were not used as a confounding element.

\subsection{Questionnaires}

The results of the questionnaires are presented in Table 2 . General fatigue was significantly reduced at discharge in the IU group compared to the CU group. This reduction was defined by MFI-20 general (IU, $n=28 ; \mathrm{CU}, n=30 ; P=0.025)$. The feeling of being rested was also significantly reduced in favor of the IU based on the statement, "I woke up fresh and rested" (IU, $n=28 ; \mathrm{CU}, n=30 ; P=0.025$ ). There were no significant differences between patients in the IU and 
Table 3

Mean/median scores at inclusion and discharge in each unit

\begin{tabular}{|c|c|c|}
\hline Questionnaires & $\begin{array}{c}\text { Inclusion score } \\
\text { Mean } \pm \mathrm{SD} / \\
\text { median (IQR) }\end{array}$ & $\begin{array}{c}\text { Discharge score } \\
\text { Mean } \pm \mathrm{SD} / \\
\text { median (IQR) }\end{array}$ \\
\hline \multicolumn{3}{|l|}{ PSQI } \\
\hline Control Unit $(n=30)$ & $7.5 \pm 4.0$ & $9.6 \pm 5.3$ \\
\hline Intervention Unit $(n=27)$ & $6.9 \pm 3.8$ & $7.9 \pm 4.1$ \\
\hline \multicolumn{3}{|l|}{ ESS } \\
\hline Control Unit $(n=30)$ & $5.5(1.0-11.0)$ & $5.5(0.0-8.0)$ \\
\hline Intervention Unit $(n=26)$ & $7.0(1.0-12.0)$ & $4.5(0.0-10.0)$ \\
\hline \multicolumn{3}{|l|}{ Rested Statement } \\
\hline Control Unit $(n=30)$ & $3.0(0.0-5.0)$ & $3.0(0.0-4.0)$ \\
\hline Intervention Unit $(n=28)$ & $4.0(0.0-5.0)$ & $4.0(1.0-5.0)$ \\
\hline \multicolumn{3}{|l|}{ MFI-20 global } \\
\hline Control Unit $(n=30)$ & $61.4 \pm 15.8$ & $61.2 \pm 16.5$ \\
\hline Intervention Unit $(n=28)$ & $55.0 \pm 17.3$ & $53.6 \pm 16.0$ \\
\hline \multicolumn{3}{|l|}{ MFI-20 activity } \\
\hline Control Unit $(n=30)$ & $15.0(4.0-18)$ & $15.5(5.0-18)$ \\
\hline Intervention Unit $(n=28)$ & $15.0(4.0-17.5)$ & $14.5(4.0-16.0)$ \\
\hline \multicolumn{3}{|l|}{ MFI-20 general } \\
\hline Control Unit $(n=30)$ & $14.0(5.0-17.0)$ & $14.5(5.0-17)$ \\
\hline Intervention Unit $(n=28)$ & $12.0(4.0-14.5)$ & $10.5(4.0-14.0)$ \\
\hline \multicolumn{3}{|l|}{ MFI-20 mental } \\
\hline Control Unit $(n=30)$ & $9.9 \pm 4.4$ & $9.5( \pm 4.3)$ \\
\hline Intervention Unit $(n=28)$ & $7.9 \pm 4.3$ & $7.5( \pm 3.8)$ \\
\hline \multicolumn{3}{|l|}{ MFI-20 motivation } \\
\hline Control Unit $(n=30)$ & $9.5(4.0-11.0)$ & $8.5(4.0-12.0)$ \\
\hline Intervention Unit $(n=28)$ & $7.0(4.0-9.5)$ & $8.0(4.0-10.0)$ \\
\hline \multicolumn{3}{|l|}{ MFI-20 physical } \\
\hline Control Unit $(n=30)$ & $15.5(6.0-18.0)$ & $15.5(4.0-18.0)$ \\
\hline Intervention Unit $(n=28)$ & $17.0(4.0-20.0)$ & $14.5(4.0-18.0)$ \\
\hline
\end{tabular}

CU regarding MFI-20 global and the four other MFI20 subgroups, sleepiness (ESS), or subjective sleep quality (PSQI). However, the scores were all in favor of the IU.

Mean/median scores at inclusion and discharge in each unit are illustrated in Table 3. These results are actual mean or median scores and therefore without adjustment for the described confounders.

The variance between the time-points indicates a significant diurnal rhythm of VAS sleepiness in both groups at inclusion and discharge. The diurnal pattern of sleepiness significantly changed during the admission in each group overall (IU: $P=0.003$; CU: $P \leq 0.0001$ ) (Table $4 \mathrm{a}, \mathrm{b}$ ). Considering the specific time-point, at midnight and 04:00 a.m., sleepiness was significantly elevated in both groups between inclusion and discharge. This diurnal pattern of sleepiness is also illustrated by the curves in Fig. 2, where sleepiness increases slightly during the first 12 hours of the day, peaks at midnight, and then decreases from midnight to dawn.

\subsection{Confounding effect of antidepressants, sleep medication and sleep apnea}

When looking at the influence of the antidepressant medication, which also were a result of the analyzes of covariance, there was significantly lower scores in the MFI-20 general in favor of patients with no antidepressant medication prescriptions compared with patients with antidepressant medication prescriptions (difference, $-28.2 \%$; $95 \%$ CI $[-41.7 \%$; $-3.0 \%] ; P=0.003)$. In the analysis of the Rested statement there was a near-significant difference between patients using and not using antidepressant medication (difference, 35\%; 95\% CI [-1.7\%; 91.8\%]; $P=0.064)$. This result indicates a confounding effect of antidepressant medication prescriptions (number of patients treated with antidepressant medication: IU, $n=16$; CU, $n=9$ ) and sleep medication (IU, $n=8$; $\mathrm{CU}, n=8)$. No confounding effect of sleep medication and sleep apnea was found in fatigue scores or subjective sleep quality scores. A total number of 33 patients had severe sleep apnea (16 IU; $17 \mathrm{CU}), 17$ 
Table 4a

Calculated variance between time-points for sleepiness at the Intervention Unit

\begin{tabular}{lcccc}
\hline Time & $\begin{array}{c}\text { Difference in score* } \\
\text { INTERVENTION UNIT }(n=20)\end{array}$ & \multicolumn{2}{c}{ IQR } & $P$ value \\
\hline INAS difference) & Lower & Upper & \\
\hline VAS sleepiness inclusion ${ }^{1}$ & - & - & - & $\mathbf{0 . 0 1 9}$ \\
08:00 a.m. & -0.703 & -1.948 & 0.542 & NS \\
Noon & -0.303 & -1.548 & 0.942 & NS \\
04:00 p.m. & -0.029 & -1.286 & 1.226 & NS \\
08:00 p.m. & 0.197 & -1.048 & 1.442 & NS \\
Midnight & 0.882 & -0.399 & 2.164 & NS \\
04:00 a.m. & 1.327 & 0.045 & 2.609 & $\mathbf{0 . 0 4 3}$ \\
Second 08:00 a.m. & 0 &. &. &. \\
VAS sleepiness discharge ${ }^{1}$ & - & - & - & $<\mathbf{0 . 0 0 0 1}$ \\
08:00 a.m. & -0.264 & -1.398 & 0.870 & NS \\
Noon & 0.247 & -0.884 & 1.379 & NS \\
04:00 p.m. & -0.303 & -1.427 & 0.821 & NS \\
08:00 p.m. & 0.458 & -0.674 & 1.589 & NS \\
Midnight & 2.375 & 1.214 & 3.536 & $<\mathbf{0 . 0 0 0 1}$ \\
04:00 a.m. & 2.198 & 1.037 & 3.359 & $\mathbf{0 . 0 0 0 3}$ \\
Second 08:00 a.m. & 0 &. &. &. \\
Variance, inclusion vs. discharge ${ }^{1}$ & - & - & - & $\mathbf{0 . 0 0 3}$ \\
08:00 a.m. & 0.227 & -0.979 & 1.432 & NS \\
Noon & 0.522 & -0.679 & 1.724 & NS \\
04:00 p.m. & -0.208 & -1.412 & 0.996 & NS \\
08:00 p.m. & 0.606 & -0.597 & 1.809 & NS \\
Midnight & 1.997 & 0.713 & 3.281 & $\mathbf{0 . 0 0 3}$ \\
04:00 a.m. & 1.636 & 0.342 & 2.930 & $\mathbf{0 . 0 1 4}$ \\
Second 08:00 a.m. & 0 &. &. &. \\
\hline
\end{tabular}

Changes between time-points for diurnal sleepiness (Proc mixed, SAS). ${ }^{1}$ Type 3 tests of fixed effects. NS = not significant. *The time-point "Second 08:00 a.m." is the reference the difference is calculated from.

Table $4 \mathrm{~b}$

Calculated variance between time-points for sleepiness at the Control Unit

\begin{tabular}{lcccc}
\hline Time & $\begin{array}{c}\text { Difference in score } \\
\text { CONTROL UNIT }(n=19)\end{array}$ & \multicolumn{2}{c}{ IQR } & $P$ value \\
\cline { 3 - 5 } (median difference) & Lower & Upper & \\
\hline VAS sleepiness inclusion ${ }^{1}$ & - & - & - & $\mathbf{0 . 0 0 1}$ \\
08:00 a.m. & -0.066 & -1.371 & 1.239 & NS \\
Noon & 0.829 & -0.476 & 2.133 & NS \\
04:00 p.m. & 0.934 & -0.371 & 2.239 & NS \\
08:00 p.m. & 0.829 & -0.476 & 2.133 & NS \\
Midnight & 2.354 & 1.037 & 3.670 & $\mathbf{0 . 0 0 0 6}$ \\
04:00 a.m. & 1.909 & 0.593 & 3.226 & $\mathbf{0 . 0 0 5}$ \\
Second 08:00 a.m. & 0 &. &. &. \\
VAS sleepiness discharge ${ }^{1}$ & - & - & - & $<\mathbf{0 . 0 0 0 1}$ \\
08:00 a.m. & -0.846 & -2.211 & 0.518 & NS \\
Noon & -0.373 & -1.737 & 0.992 & NS \\
04:00 p.m. & -0.320 & -1.685 & 1.045 & NS \\
08:00 p.m. & 0.714 & -0.692 & 2.120 & NS \\
Midnight & 2.525 & 1.148 & 3.902 & $\mathbf{0 . 0 0 0 4}$ \\
04:00 a.m. & 2.004 & 0.583 & 3.426 & $\mathbf{0 . 0 0 6}$ \\
Second 08:00 a.m. & 0 &. &. &. \\
Variance, inclusion to discharge & - & - & - & $<\mathbf{0 . 0 0 0 1}$ \\
08:00 a.m. & - & -2.429 & 0.382 & NS \\
Noon & -1.024 & -1.988 & 0.855 & NS \\
04:00 p.m. & -0.567 & -1.941 & 0.909 & NS \\
08:00 p.m. & -0.516 & -0.923 & 1.994 & NS \\
Midnight & 0.535 & 0.795 & 3.808 & $\mathbf{0 . 0 0 3}$ \\
04:00 a.m. & 2.302 & 0.252 & 3.314 & $\mathbf{0 . 0 2 3}$ \\
Second 08:00 a.m. & 1.783 &. &. &. \\
\hline
\end{tabular}

Changes between time-points for diurnal sleepiness (Proc mixed, SAS). ${ }^{1}$ Type 3 tests of fixed effects. NS $=$ not significant. *The time-point "Second 08:00 a.m." is the reference the difference is calculated from. 

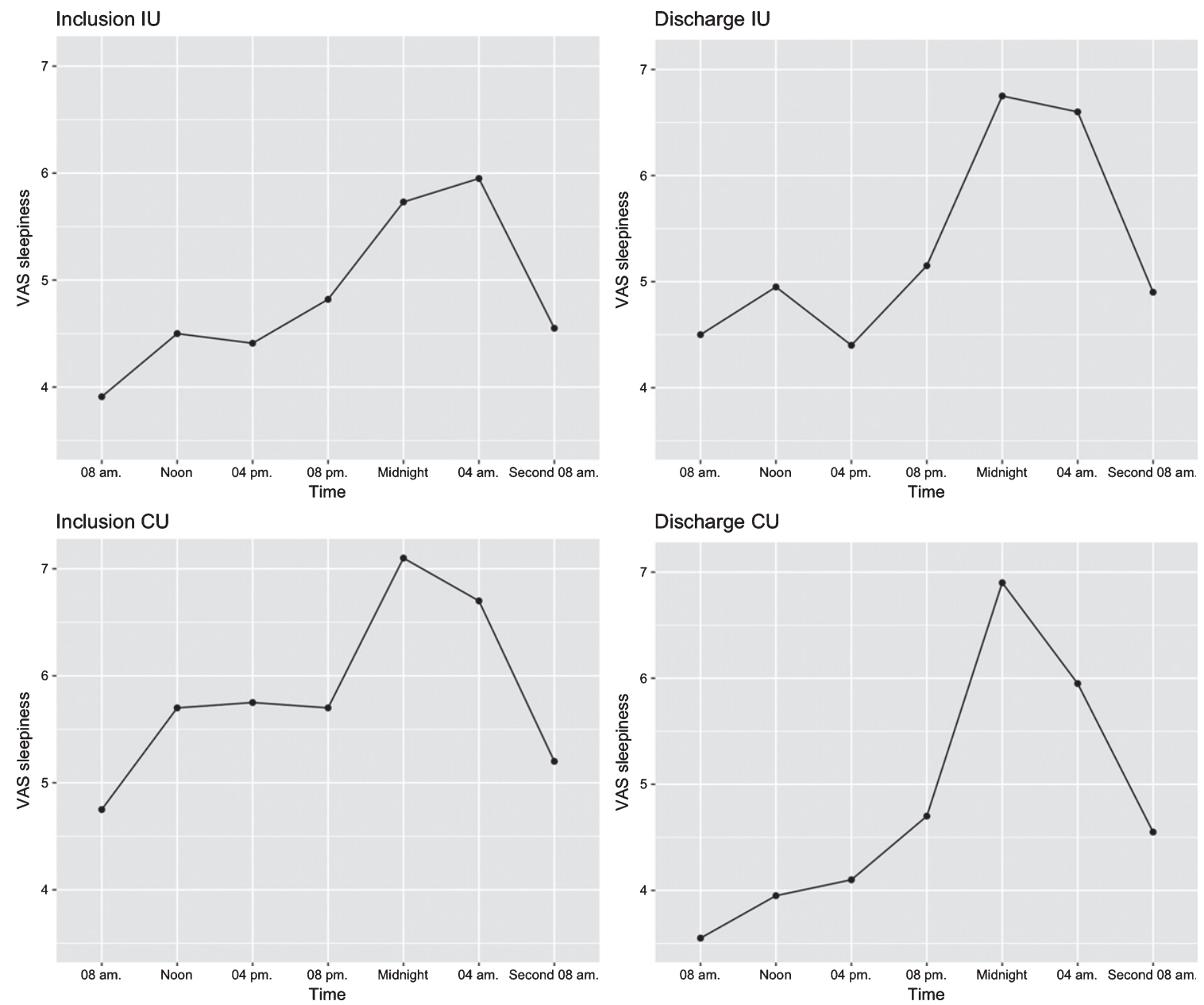

Fig. 2. Mean VAS scores at all seven time-points. Mean scores at all seven timepoints subdivided into groups: inclusion and discharge (IU, $n=20 ; \mathrm{CU}, n=19$ ).

had moderate (8 IU; 9 CU), 6 had mild (4 IU; 2 CU) and 4 patients had no sleep apnea (2 IU; 2 CU). Two patients were treated with CPAP, one at each unit.

\subsection{Interactions}

No significant interaction among sleep medication, antidepressant medication, and sleep apnea was found.

\subsection{Correlation between measured fatigue and depression}

Significant correlation was found by regression analysis between scores of MFI-20 and Major depression inventory scale (MDI) in time of inclusion and discharge (Inclusion: $x=0.94 \mathrm{y}+0.13, P=0.0002$; Discharge: $x=1.30 \mathrm{y}-1.18, P=<0.0001)$.

\section{Discussion}

The current study has yielded the first findings of a significant effect of naturalistic lighting on poststroke fatigue and feeling of waking up rested, with no effects on subjective sleep quality or sleepiness.

A significant reduction was found in the subscore MFI-20 General. There was an improvement in favor of the IU regarding the MFI-20 global and all subscores, activity, mental, motivation, and physical domains, although not significantly. Because most stroke patients have a strong motivation for rehabilitation, this factor may have interfered with their response to mental and motivational questions (Maclean, Pound, Wolfe, \& Rudd, 2000; Mayo, Fellows, Scott, Cameron, \& Wood-Dauphinee, 2009). We did not expect a better outcome in physical rehabilitation between groups in this study, thus it is not 
surprising that no significant difference was found regarding physical fatigue. The mental part reflects a cognitive element. The prevalence of cognitive impairment is severe after stroke (Mohd Zulkifly, Ghazali, Che Din, Singh, \& Subramaniam, 2016) and remains highly prevalent several years after the first stroke (Patel, Coshall, Rudd, \& Wolfe, 2003), which may explain the lack of significant improvement in these subscores. Activity scores may be falsely low and inaccurate because the patients in most cases did not have any influence on training frequency; the training was implemented in the daily plans and not at the patient's initiative. MFI-20 focus only on the actual activity level during the day and not the desire for activity. This factor may also have influenced the outcome but it is reasonable to suggest that the absence of significant differences in these subscores and thereby MFI-20 global could also be the result of a lack of power.

The Rested Statement, "I woke up fresh and rested," was included because it is an easily understandable, simple, and informative statement that provides information regarding how rested a patient feels. It was significantly improved in favor of the IU. The fact that the feeling of rest may reflect both sleep quality and the feeling of fatigue could be the reason for the significant outcome, but additional studies should elaborate on this.

The combination of depression and fatigue is a known phenomenon in severe illness, especially in stroke (Hinkle et al., 2017; Naess, Lunde, \& Brogger, 2012; Snaphaan, van der Werf, \& de Leeuw, 2010). Depression as a sequela after stroke is like fatigue an frequent complication and is observed in approx. 30\% of post stroke patients (Everson, Roberts, Goldberg, \& Kaplan, 1998). Comparison of depressive mood between the two units in this cohort is to be published elsewhere. In this study, as expected, we found that the depressive scores correlated significantly with the fatigue scores. Depression and fatigue have a known influence on each other, and it cannot be ruled out that the observed effect on fatigue represents an improvement in depressive mood. Despite the correlation between depression and fatigue, fatigue can be present without sign of depression and vice versa indicating different pathophysiologies as the lack of effect of antidepressants on fatigue also indicates (Choi-Kwon, Choi, Kwon, Kang, \& Kim, 2007). Fatigue may therefore not be just a sign of depression, but also a frequent independent symptom that is observed as part of mental stroke complications (Bourgeois, Hilty, Chang, Wineinger,
$\&$ Servis, 2004). The known positive effect blue light has on depressive mood (Kopp et al., 2016; Lam et al., 2016) may therefore not be the whole explanation for the reduction in fatigue score in the intervention group in this study. Still, it may be assumed that the depressive symptoms in these patients have a negative influence on the measured fatigue. This influence is impossible to measure and include as a confounder because of the interactions these symptoms have in stroke patients. Based on the found correlation between fatigue and depression, along with the fact that MFI-20 shows similarity with questionnaires concerning mood, antidepressant medication was chosen to be adjusted for in the calculation. The difference between groups regarding number of patients prescribed antidepressant medication, with more prescriptions in the IU, was due to the statistical adjustment, not an element that could affect the outcome.

No studies have investigated naturalistic light as an intervention for post-stroke fatigue. However, bright light seems to reduce fatigue in patients with conditions known to present fatigue as sequelae (Ancoli-Israel et al., 2011; Sinclair et al., 2014). Even though fatigue is thought to be regulated by the circadian clock, a direct response to the light could also play a role in fatigue reduction. Cortisol levels increase in response to the change from dim light to bright light exposure in the morning, but not in the afternoon or night (Leproult, Colecchia, L'Hermite-Balériaux, \& Van Cauter, 2001). This physical response to the natural light rhythm induces the arousal in the morning and may hypothetical reduce the feeling of fatigue in a more direct way in the patients admitted at the IU.

Treating post-stroke fatigue is a challenge in clinical practice (Hinkle et al., 2017). To date, no non-pharmacological intervention has been identified that could significantly prevent or decrease fatigue after stroke (Hinkle et al., 2017; Wu et al., 2015). Thus, this study is the first to present a significant reduction in fatigue in post-stroke patients exposed to a non-pharmacological treatment.

We found no improvement in subjective sleep quality measured by PSQI between the two groups. The stroke-induced motor impairments might lead to less stimulation of the circadian structures regulating the sleep-wake cycle, which could trigger rhythm desynchronization and sleep difficulties. The severity of the impaired sleep quality may have overcome an eventual effect of the installed naturalistic light, and it is reasonable to believe that light does not affect sleep 
quality but only sleep rhythm control, as the few known studies concerning subjective sleep quality have not shown clear improvement using bright light as an intervention (De Rui et al., 2014; Kobayashi et al., 2001; Perras et al., 2007). A review and metaanalysis from 2014 investigated bright-light therapy vs control regarding total sleep duration, sleep efficiency, and night-time awaking and identified a similar lack of effect of bright light (Forbes, Blake, Thiessen, Peacock, \& Hawranik, 1996). We did measure objective sleep quality with polysomnography and actigraphy, but we have chosen to present these data in a separate article because of the severity of the findings in objective sleep quality. The lack of improvement in subjective sleep quality at the time of discharge is in line with the notion, mention in the introduction section, of fatigue as a different pathology than sleepiness and therefore not necessarily related to sleep quality and sleepiness.

There was no significant difference between groups regarding sleepiness (ESS scores). The diurnal changes in sleepiness between inclusion and discharge measured by VAS sleepiness seemed similar between the groups, which support the lack of significant differences found in ESS. The lack of a significant difference in sleepiness between the two groups may be related to the lack of improvement in sleep quality due to the brain damage but also due to the moderate to severe sleep apnea measured to be present in this patient cohort. The light has no assumed theoretical physical impact in the mechanism of sleep apnea, which may be one of the explanations behind the nonsignificant outcome.

\subsection{Limitations and strengths}

Not all participants could answer all questionnaires. It was not possible to identify who would be able to complete the questionnaires prior to inclusion, so we chose to include all eligible patients despite complications that could make them unable to do so, inducing a risk of dropouts. Unawareness was the main reason for dropout after inclusion. IU still had a higher National Institutes of Health Stroke Scale (NIHSS) score at discharge (non-significant), illustrating that stroke severity had no clear influence on the higher dropout rates in the IU.

The randomization followed the normal procedure for distribution of patients to the two rehabilitations units. It would have been optimal to have had totally blinded randomizing; however, this process was not feasible in the clinical setting because of the visible intervention. The conditions in the two rehabilitation units were equal regarding staff profession, interior, form, and size. Information on all bed positions (two beds: window bed and away-from-window bed) were collected during the study. However, all patients were placed at the window at the end of the stay because of natural rotation in the units. Therefore, no differences in bed positions among patients were found, so bed positions were not included as a confounding element. The locations of the two units were not equal regarding angle to the sun, but a measurement of the incoming light from the sun between the two units revealed no significant differences (West et al., 2017), assuming that levels above 200 lux would stimulate the circadian center (Andersen, Mardaljevic, \& Lockley, 2012). There was no significant difference in daylight exposure at the window bed across the year, as presented in West et al. Fig. 4a and $4 \mathrm{~b}$ (West et al., 2017). A difference in daylight exposure between IU and $\mathrm{CU}$ at the door bed was observed, but all illuminance levels fell below the required level of 200 lux D55 equivalent light that would stimulate the circadian center (Andersen et al., 2012). Because of that, we choose to claim that the difference in daylight exposure between the units for the door beds was not significant and did not favor the IU.

There was a significant difference between the two groups in the duration of the inclusion period, and thus the length of the inclusion period was chosen as a confounding variable in the analysis, to eliminate any possible influence on the outcome. The main explanation behind differences in admission length might be that it was not the same rehabilitation team that dictated the time of discharge at the two units. Furthermore, the higher NIHSS score in the IU may also have influenced the longer admission time; however, no other differences were found between the two groups. Because of the real clinical setting, it is relevant to reflect about possible confounders. However, despite being two separate units, we believe, due to the RCT setup and the fact that the units are run similarly, that we have included the most relevant confounders after many reflections and deep insight into the daily life at the two units especially since the first and last author have worked as clinical doctors at the two units before study inclusion. However, further RCT studies in a real clinical environment should explore this.

Only patients in treatment with sleep and/or antidepressant medication at discharge were included in 
the covariance analysis as prescriptions in the interim period between these time points were estimated to be of shorter duration and therefore of limited impact on fatigue and sleepiness. In addition to this, it was not clinical practice to discontinuing antidepressant after prescription except for occurred side effects, why short prescription period may have been rare or not present.

To summarize the choice of confounders in this study, were following confounders chose to be included in the statistical analyses; antidepressant medicine, sleep medicine and length of inclusion. Confounders which were found not relevant to include were sleep apnea, bed positions and MEQ.

In this study, several tests were performed, increasing the risk of false positive results. All tests favored the intervention. If a penalty for multiple comparison had been used e.g. Bonferroni correction, there would be a risk of false negative results, missing out on potentially important effects of the light and thereby maybe prevent future studies from investigating the effects of naturalistic lighting. However, a Benjaminini-Hochberg procedure was conducted with a false discovery rate of 0.15 resulting in survived significance for the two significant $p$-values in Table 2.

The strengths of this study were the real clinical setting and two equal units in the same institution. Therefore, we believe that our data can be directly transferred to clinical everyday life. Whether it has any clinical value for the patients after discharge must be elaborated in further studies. However, this study is part of an exploratory investigation in a relatively new scientific area. More specific studies are therefore required to address the effects of naturalistic light on sleep quality and fatigue.

\section{Conclusions}

The aim of this study was to test the effects of naturalistic light on fatigue and subjective sleep quality in a real hospital setting. We showed that patients exposed to naturalistic lighting during admission had decreased fatigue compared with patients exposed to standard indoor lighting, with no effect on subjective sleep and sleepiness. Naturalistic light could, to our knowledge, be the first non-pharmacological treatment shown to decrease fatigue in hospitalized post-stroke patients. But the exploratory design in this study requires further studies to address this effect.

\section{Acknowledgments}

We are deeply grateful to the stroke patients for participation in the study. We thank service manager Svend Morten Christiansson and architect Maj Lis Brunsgård Seligmann from the Service Center Rigshospitalet for initiating the focus on the importance of lighting and make it possible to install naturalistic light on an entire hospital ward. We thank the company Chromaviso and especially master in optical engineering, Torben Skov Hansen for always being available for technical questions regarding the light setup. Thanks to Nina V. Grønberg, MD, for help during periods of high work pressure. Thanks to the health staff at the entire stroke department, Rigshospitalet, for their engagement and professionalism through their provided support and logistical assistance during the project period.

\section{Conflict of interest}

All authors have completed the Unified Competing Interest form and declare no support from any organization for the submitted work, no financial relationships with any organizations that might have an interest in the submitted, and no other relationships or activities that could appear to have influenced the submitted work.

Chromaviso delivered the light installation and had no influence on the study design or interpretation of the results.

This study received research grant support from the Market Development Foundation Denmark and support from the involved departments. The funders of the study had no role in the study design, data collection, data analysis, data interpretation, or writing.

\section{References}

Allgaier, A. -K., Kramer, D., Saravo, B., Mergl, R., Fejtkova, S., \& Hegerl, U. (2013). Beside the Geriatric Depression Scale: The WHO-Five Well-being Index as a valid screening tool for depression in nursing homes. International Journal of Geriatric Psychiatry, 28(11), 1197-1204.

Ancoli-Israel, S., Gehrman, P., Martin, J. L., Shochat, T., Marler, M., Corey-Bloom, J., \& Levi, L. (2003). Increased light exposure consolidates sleep and strengthens circadian rhythms in severe Alzheimer's disease patients. Behavioral Sleep Medicine, 1(1), 22-36.

Ancoli-Israel, S., Liu, L., Rissling, M., Natarajan, L., Neikrug, A. B., Palmer, B. W., Mills, P. J., et al. (2014). Sleep, fatigue, 
depression, and circadian activity rhythms in women with breast cancer before and after treatment: A 1-year longitudinal study. Supportive Care in Cancer, 22(9), 2535-2545.

Ancoli-Israel, S., Rissling, M., Neikrug, A., Trofimenko, V., Natarajan, L., Parker, B. A., Lawton, S., et al. (2011). Light treatment prevents fatigue in women undergoing chemotherapy for breast cancer. Supportive Care in Cancer, 20(6), 12111219.

Andersen, M., Mardaljevic, J., \& Lockley, S. (2012). A framework for predicting the non-visual effects of daylight - Part I: Photobiology- based model. Lighting Research and Technology, 44(1), 37-53.

Bernhofer, E. I., Higgins, P. A., Daly, B. J., Burant, C. J., \& Hornick, T. R. (2013). Hospital lighting and its association with sleep, mood and pain in medical inpatients. Journal of Advanced Nursing, 70(5), 1164-1173.

Berry, R. B., Budhiraja, R., Gottlieb, D. J., Gozal, D., CConrad, I., Kapur, V. K., Marcus, C. L., et al. (2012). Rules for scoring respiratory events in sleep: Update of the 2007 AASM manual for the scoring of sleep and associated events. Journal of Clinical Sleep Medicine: JCSM : Official Publication of the American Academy of Sleep Medicine, 1-23.

Bourgeois, J. A., Hilty, D. M., Chang, C. H., Wineinger, M. A., \& Servis, M. E. (2004). Poststroke neuropsychiatric illness: An integrated approach to diagnosis and management. Current treatment options in neurology, 6(5), 403-420.

Chen, Y. -K., Qu, J. -F., Xiao, W. -M., Li, W. -Y., Weng, H. -Y., Li, W., Liu, Y. -L., et al. (2015). Poststroke fatigue: Risk factors and its effect on functional status and health-related quality of life. International Journal of Stroke, 10(4), 506-512.

Cho, J. R., Joo, E. Y., Koo, D. L., \& Hong, S. B. (2013). Let there be no light: The effect of bedside light on sleep quality and background electroencephalographic rhythms. Sleep Medicine, 14(12), 1422-1425.

Choi-Kwon, S., Choi, J., Kwon, S. U., Kang, D. -W., \& Kim, J. S. (2007). Fluoxetine Is not effective in the treatment of poststroke fatigue: A double-blind, placebo-controlled study. Cerebrovascular Diseases, 23(2-3), 103-108.

Cumming, T. B., Packer, M., Kramer, S. F., \& English, C. (2016). The prevalence of fatigue after stroke: A systematic review and meta-analysis. International Journal of Stroke, 11(9), 968-977.

Czeisler, C. A., Weitzman, E. D., Moore-Ede, M. C., Zimmerman, J. C., \& Knauer, R. S. (1980). Human sleep: Its duration and organization depend on its circadian phase. Science (New York, NY), 210(4475), 1264-1267.

De Doncker, W., Dantzer, R., Ormstad, H., \& Kuppuswamy, A. (2017). Mechanisms of poststroke fatigue. Journal of Neurology, Neurosurgery, and Psychiatry, jnnp-2017-316007-8.

De Rui, M., Middleton, B., Sticca, A., Gatta, A., Amodio, P., Skene, D. J., \& Montagnese, S. (2014). Sleep and circadian rhythms in hospitalized patients with decompensated cirrhosis: Effect of light therapy. Neurochemical Research, 40(2), 284-292.

Dzierzewski, J. M., Fung, C. H., Jouldjian, S., Alessi, C. A., Irwin, M. R., \& Martin, J. L. (2014). Decrease in daytime sleeping is associated with improvement in cognition after hospital discharge in older adults. Journal of the American Geriatrics Society, 62(1), 47-53.

Everson, S. A., Roberts, R. E., Goldberg, D. E., \& Kaplan, G. A. (1998). Depressive symptoms and increased risk of stroke mortality over a 29-year period. Archives of internal medicine, $158(10), 1133-1138$.
Feigin, V. L., Mensah, G. A., Norrving, B., Murray, C. J. L., Roth, G. A., \& GBD 2013 Stroke Panel Experts Group. (2015). Atlas of the global burden of stroke (1990-2013): The GBD 2013 study. Neuroepidemiology, 45(3), 230-236.

Forbes, D., Blake, C. M., Thiessen, E. J., Peacock, S., \& Hawranik, P. (1996). Light therapy for improving cognition, activities of daily living, sleep, challenging behaviour, and psychiatric disturbances in dementia. (D. Forbes, Ed.) (pp. 1-64). Chichester, UK: John Wiley \& Sons, Ltd.

Goldman, S. E., Stone, K. L., Ancoli-Israel, S., Blackwell, T., Ewing, S. K., Boudreau, R., Cauley, J. A., et al. (2007). Poor sleep is associated with poorer physical performance and greater functional limitations in older women. Sleep, 30(10), 1317-1324

Hermann, D. M., S. M. B. C. (2004). Sleep-wake disorders and stroke, 1-5.

Hermann, D. M., \& Bassetti, C. L. (2009). Sleep-related breathing and sleep-wake disturbances in ischemic stroke. Neurology, 73(16), 1313-1322.

Hermann, D. M., \& Bassetti, C. L. (2016). Role of sleep-disordered breathing and sleep-wake disturbances for stroke and stroke recovery. American Academy of Neurology, 87(13), 14071416.

Hinkle, J. L., Becker, K. J., Kim, J. S., Choi-Kwon, S., Saban, K. L., McNair, N., \& Mead, G. E. (2017). Poststroke fatigue: Emerging evidence and approaches to management: A scientific statement for healthcare professionals from the american heart association. Stroke, 48(7), e159-e170.

Horne, J. A., \& Ostberg, O. (1976). A self-assessment questionnaire to determine morningness-eveningness in human circadian rhythms. International Journal of Chronobiology, 4(2), 97-110.

Ingles, J. L., Eskes, G. A., \& Phillips, S. J. (1999). Fatigue after stroke. Archives of Physical Medicine and Rehabilitation, 80(2), 173-178.

Jennum, P., Iversen, H. K., Ibsen, R., \& Kjellberg, J. (2015). Cost of stroke: A controlled national study evaluating societal effects on patients and their partners. BMC Health Services Research, 15(1), 181-110. BioMed Central.

Johns, M. W. (1991). A new method for measuring daytime sleepiness: The Epworth sleepiness scale. Sleep, 14(6), 540-545.

Kobayashi, R., Fukuda, N., Kohsaka, M., Sasamoto, Y., Sakakibara, S., Koyama, E., Nakamura, F., et al. (2001). Effects of bright light at lunchtime on sleep of patients in a geriatric hospital I. Psychiatry and Clinical Neurosciences, 55(3), 287-289.

Kopp, B. T., Hayes, D., Jr, Ghera, P., Patel, A., Kirkby, S., Kowatch, R. A., \& Splaingard, M. (2016). Pilot trial of light therapy for depression in hospitalized patients with cystic fibrosis. Journal of Affective Disorders, 189, 164-168.

Lam, R. W., Levitt, A. J., Levitan, R. D., Michalak, E. E., Cheung, A. H., Morehouse, R., Ramasubbu, R., et al. (2016). Efficacy of bright light treatment, fluoxetine, and the combination in patients with nonseasonal major depressive disorder. JAMA Psychiatry, 73(1), 56-58.

Leproult, R., Colecchia, E. F., L'Hermite-Balériaux, M., \& Van Cauter, E. (2001). Transition from dim to bright light in the morning induces an immediate elevation of cortisol levels. The Journal of Clinical Endocrinology and Metabolism, 86(1), 151-157.

Lu, J., Greco, M. A., Shiromani, P., \& Saper, C. B. (2000). Effect of lesions of the ventrolateral preoptic nucleus on NREM and 
REM sleep. The Journal of Neuroscience: The Official Journal of the Society for Neuroscience, 20(10), 3830-3842.

Lucas, R. J., Peirson, S. N., Berson, D. M., Brown, T. M., Cooper, H. M., Czeisler, C. A., Figueiro, M. G., et al. (2014). Measuring and using light in the melanopsin age. Trends in Neurosciences, 37(1), 1-9.

Maclean, N., Pound, P., Wolfe, C., \& Rudd, A. (2000). Qualitative analysis of stroke patients' motivation for rehabilitation. $B M J$, 321(7268), 1051-1054.

Mayo, N. E., Fellows, L. K., Scott, S. C., Cameron, J., \& WoodDauphinee, S. (2009). A longitudinal view of apathy and its impact after stroke. Stroke, 40(10), 3299-3307. American Heart Association, Inc.

Mead, G. E., Graham, C., Dorman, P., Bruins, S. K., Lewis, S. C., Dennis, M. S., Sandercock, P. A. G., et al. (2011). Fatigue after stroke: Baseline predictors and influence on survival. Analysis of data from UK patients recruited in the international stroke trial. (G. Malaga, Ed.) PLoS ONE, 6(3), e16988-7. Public Library of Science.

Mishima, K., Okawa, M., Hishikawa, Y., Hozumi, S., Hori, H., \& Takahashi, K. (1994). Morning bright light therapy for sleep and behavior disorders in elderly patients with dementia. Acta psychiatrica Scandinavica, 89(1), 1-7.

Mohd Zulkifly, M. F., Ghazali, S. E., Che Din, N., Singh, D. K. A., \& Subramaniam, P. (2016). A review of risk factors for cognitive impairment in stroke survivors. The Scientific World Journal, 2016(3), 1-16.

Naess, H., Lunde, L., \& Brogger, J. (2012). The triad of pain, fatigue and depression in ischemic stroke patients: The bergen stroke study. Cerebrovascular Diseases, 33(5), 461-465.

Patel, M., Coshall, C., Rudd, A. G., \& Wolfe, C. D. (2003). Natural history of cognitive impairment after stroke and factors associated with its recovery. Clinical Rehabilitation, 17(2), 158-166.

Perras, B., Meier, M., \& Dodt, C. (2007). Light and darkness fail to regulate melatonin release in critically ill humans. Intensive Care Medicine, 33(11), 1954-1958.
Satlin, A., Volicer, L., Ross, V., Herz, L., \& Campbell, S. (1992). Bright light treatment of behavioral and sleep disturbances in patients with Alzheimer's disease. The American Journal of Psychiatry, 149(8), 1028-1032.

Siengsukon, C. F., \& Boyd, L. A. (2009). Sleep to learn after stroke: Implicit and explicit off-line motor learning. Neuroscience Letters, 451(1), 1-5.

Sinclair, K. L., Ponsford, J. L., Taffe, J., Lockley, S. W., \& Rajaratnam, S. M. W. (2014). Randomized controlled trial of light therapy for fatigue following traumatic brain injury. Neurorehabilitation and Neural Repair, 28(4), 303-313.

Snaphaan, L., van der Werf, S., \& de Leeuw, F. E. (2010). Time course and risk factors of post-stroke fatigue: A prospective cohort study. European Journal of Neurology, 18(4), 611-617.

Thapan, K., Arendt, J., \& Skene, D. J. (2001). An action spectrum for melatonin suppression: Evidence for a novel non-rod, non-cone photoreceptor system in humans. The Journal of Physiology, 535(Pt 1), 261-267.

van de Port, I. G. L., Kwakkel, G., Schepers, V. P. M., Heinemans, C. T. I., \& Lindeman, E. (2007). Is fatigue an independent factor associated with activities of daily living, instrumental activities of daily living and health-related quality of life in chronic stroke? Cerebrovascular Diseases, 23(1), 40-45.

Venkateshiah, S. B. (2012). Sleep and Sleep Disorders in the Hospital. CHEST Journal, 141(5), 1337-1339.

Wakamura, T., \& Tokura, H. (2001). Influence of bright light during daytime on sleep parameters in hospitalized elderly patients. Journal of Physiological Anthropology and Applied Human Science, 20(6), 345-351.

West, A., Jennum, P., Simonsen, S. A., Sander, B., Pavlova, M., \& Iversen, H. K. (2017). Impact of naturalistic lighting on hospitalized stroke patients in a rehabilitation unit: Design and measurement. Chronobiology International, 34(6), 687-697.

Wu, S., Kutlubaev, M. A., Chun, H.-Y. Y., Cowey, E., Pollock, A., Macleod, M. R., Dennis, M., et al. (2015). Interventions for post-stroke fatigue. Cochrane Database of Systematic Reviews (Online), (7), CD007030. 